\title{
Numerical Analysis and Computational Methods of Heat Flow in Fire Compartment
}

\author{
MASAHIRO MORITA \\ Department of Applied Mathematics \\ Faculty of Science \\ Science University of Tokyo \\ 1-3 Kagurazaka, Shinjuku-ku \\ Tokyo 162, Japan
}

\section{MASAYUKI HIROTA}

Fire Safety Group

Department of Planning Engineering

Institute of Technology

Shimizu Construction Co., Ltd

3-4-17 Etcgujima, Kohtoh-ku, Tokyo 135, Japan

\author{
YASUSHI OKA \\ Department of Architecture \\ Faculty of Science and Technology \\ Science University of Tokyo \\ 2641 Yamazaki, Noda-shi \\ Chiba 278, Japan
}

\begin{abstract}
Theoretical and experimental numerical analysis have proposed the capable of being executed computational finite difference method for fire induced natural convective heat flow using the viscous heat conductive compressible fluid with $\mathrm{K}-\epsilon$ model in the fire compartment. Because two-point upwind difference scheme give the numerical viscosity, the computational results are different from the approximate solutions at the large velocity. The practical stability and the truncation errors for computing finite difference equations approximating fire governing equations have been introduced by theoretical numerical analysis. The sensitivities of numerical solutions have been evaluated by the theoretical and experimental numerical analysis. As the results of numerical experiments we proposed that the reasonable time interval and space mesh size are chosen considering the CPU time. Furthermore we have introduced the $R e^{*}$ for the equation of motion or $P e^{*}$ for the equation of energy. We proposed that the values of $R e^{*}$ and $P e^{*}$ indicate the trust in the approximate solutions in consequence of the numerical experiments.
\end{abstract}

\section{KEYWORDS}

Fire simulation; Compartment fire; Compressible fluid; Stability; Spurious oscillation; Truncation error; Effective maxinum cell $R e^{*}$; Effective maximum cell $P e^{*}$; $\mathrm{K}-\epsilon$ model

\section{INTRODUCTION}

The numerical computations of a natural convective flow have been studied mainly in fluid dynamics [1]. In applied mathematics the theoretical and numerical analysis of Navier-Stokes (N-S) equation have been investigated [2]. Ladyzhenskaya [2] proposed that the unique solution and the existence of analytical solution of 
N-S equation for the incompressible fluid flow are not guaranteed in high Reynolds number and only guaranteed at small $R e$ number (less than about 100) at the initial and boundary condition. The mathematical analysis for the compressible fluid flow does not be reported yet. None the less, the computer simulations for the field model applied to the fire phenomena have been reported by Hasemi [3]. However it is necessary to investigate the methods of numerical solution of the non-linear parabolic partial difference equations which are the basic governing fire equations.

Because most workers using numerical methods for the convection terms in the governing equations have adopted two-point upwind difference scheme, the computational results do not give us the approximate solution because numerical viscosity is left out of consideration. Furthermore there is need to know how the truncation errors are dependence upon the time and space meshes in a fire problem influence the numerical solution.

In this paper, we have conducted calculations with several numerical computational finite difference methods for fire induced heat flow in the fire compartment using viscous heat-conductive compressible fluid (K-e model) and have made a comparion with the computational results. Since numerical experiments are a difficult computational problem requiring considerable computer power, the problem was tackled using a super computer. We have also investigated the sensitivities of the numerical solutions with the time and space meshes by using numerical experiments, and investigated the stabilities of computational scheme.

\section{GOVERNING EQUATION}

Let us consider a series of the governing equations of the turbulent natural con-

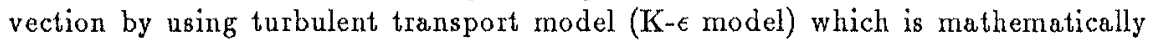
obtained by Reynolds decomposition in a fire compartment [3]. The well known field equations governing the thermophysical and thermochemical dynamics, and heat/mass transfer of a turbulent fluid are described, in principle, by the following set using two-dimensional Cartesian coordinate system.

[1] Equation of Continuity

$$
\frac{\partial \bar{\rho}}{\partial t}+\frac{\partial \rho \bar{u}_{j}}{\partial x_{j}}=0 \quad(j=1,2)
$$

or

$$
\frac{\partial^{2} \bar{P}}{\partial x_{i}^{2}}=\frac{\partial^{2} \bar{\rho}}{\partial t^{2}}+\frac{\partial^{2}}{\partial x_{i} \partial x_{j}}\left\{\bar{r}_{i j}+K\left(\frac{\partial \rho \bar{u}_{j}}{\partial x_{i}}+\frac{\partial \bar{u}_{i}}{\partial x_{j}}\right)-\bar{\rho} \bar{u}_{i} \bar{u}_{j}\right\}-g \frac{\partial \bar{\rho}}{\partial x_{2}} \quad(i, j=1,2)
$$

[2] Equation of Motion

$$
\frac{\partial \overline{\rho u_{i}}}{\partial t}+\frac{\partial \tilde{\rho} \bar{u}_{i} \bar{u}_{j}}{\partial x_{j}}=-\frac{\partial \bar{P}}{\partial x_{i}}+\frac{\partial}{\partial x_{i}}\left\{\bar{r}_{i j}+K\left(\frac{\partial \overline{\rho u_{j}}}{\partial x_{i}}+\frac{\partial \rho \bar{u}_{i}}{\partial x_{i}}\right)\right\}-\delta_{i 2} \bar{\rho} g \quad(i, j=1,2)
$$


[3] Equation of Energy

$$
\begin{aligned}
& \frac{\partial \bar{\rho} \bar{h}}{\partial t}+\frac{\partial \bar{h}_{\rho} \bar{u}_{j}}{\partial x_{j}}=\frac{\partial}{\partial x_{j}}\left(\lambda_{\theta} \frac{\partial \bar{\theta}}{\partial x_{j}}+\bar{\rho} K \frac{\partial \bar{h}}{\partial x_{j}}\right)+\bar{Q} \quad(j=1,2) \\
& \vec{h}=c_{p} \bar{\theta}
\end{aligned}
$$

[4] Equation of State

$$
\bar{P}=\bar{\rho} R \bar{\theta}
$$

[5] Transport Equation of Turbulent Energy

$$
\begin{aligned}
\frac{\partial \bar{\rho} \bar{q}}{\partial t}+\frac{\partial \bar{q} \rho \bar{u}_{j}}{\partial x_{j}}=\frac{\partial}{\partial x_{j}} & \left(\bar{\rho} K \frac{\partial \bar{q}}{\partial x_{j}}\right)+\mu \frac{\partial^{2} \bar{q}}{\partial x_{j}^{2}}+g K \frac{\partial \bar{\rho}}{\partial x_{2}}-\tilde{\rho} \bar{\epsilon} \\
& +K \frac{\partial \bar{u}_{i}}{\partial x_{j}}\left(\frac{\partial \bar{\rho}_{j}}{\partial x_{i}}+\frac{\partial \bar{\rho}_{i}}{\partial x_{j}}\right) \quad(i, j=1,2)
\end{aligned}
$$

[6] Eddy Viscosity and Energy Decay Rate

$$
\left\{\begin{array}{l}
K=c \bar{q}^{2} / \bar{\epsilon} \\
\bar{\epsilon}=c \bar{q}^{3 / 2} / l
\end{array}\right.
$$

Where $\bar{\rho}$ is density of fluid; $x$ and $y$ are spatial coordinate, horizontal and vertical direction; $\bar{u}$ and $\bar{v}$ are velocity, $x$-and $y$-direction; $t$ is time; $K$ is eddy viscosity coefficient; $\delta$ is Kronecker delta; $g$ is acceleration of gravity; $\mu$ is dynamic viscosity; $c_{p}$ is heat capacity; $\bar{\theta}$ is temperature; $\bar{q}$ is turbulent energy; $\bar{\epsilon}$ is energy decay rate; $\lambda_{\theta}$ is thermal conductivity; $\vec{h}$ is enthalpy; $\vec{P}$ is pressure; $\tilde{Q}$ is generation of energy; $l$ is Prandtl's length; $R$ is gas constant; $\tilde{\tau}$ is viscosity stress.

\section{NUMERICAL COMPUTATIONAL METHOD}

We have only discussed the equation of energy, which is the non-linear parabolic 2nd order partial differential equation, in the governing equation because the other equations will be able to deal with the same manner. The energy equation is represented by using rectangular coordinate systern; $x, y$ and $t$. The velocities, $x$ - and $\mathrm{y}$-direction, are denoted $\tilde{u}$ and $\vec{v}$ respectively. The energy equation is transformed by Reynolds stress [3] as follows;

[Equation of energy]

$$
\begin{aligned}
\frac{\partial \bar{\theta}}{\partial t}+\vec{u} \frac{\partial \bar{\theta}}{\partial x}+\bar{v} \frac{\partial \bar{\theta}}{\partial y} & =\left(\frac{\lambda_{\theta}}{\bar{\rho} c_{p}}+K\right)\left\{\frac{\partial^{2} \bar{\theta}}{\partial x^{2}}+\frac{\partial^{2} \bar{\theta}}{\partial y^{2}}\right\} \\
& +\left\{2 \frac{K}{\bar{\rho}} \frac{\partial \bar{\rho}}{\partial x}+\frac{\partial K}{\partial x}\right\} \frac{\partial \bar{\theta}}{\partial x}+\left\{2 \frac{K}{\bar{\rho}} \frac{\partial \bar{\rho}}{\partial y}+\frac{\partial K}{\partial y}\right\} \frac{\partial \bar{\theta}}{\partial y}
\end{aligned}
$$

where no generation of internal heat is adapted $\bar{Q}=0$. 
For the purpose of obtaining the approximate solution of the energy equation, let $\Delta x, \Delta y$ and $\Delta t$ be small increments of variables $x, y$ and $t$; where $\Delta x=L / I$ and $\Delta y=H / J, I$ and $J$ being integers, and $L$ and $H$ being length and height of the domain respectively. The set of point in $x, y, t$-plane given by $x=i \Delta x$, $y=j \Delta y$ and $t=n \Delta t$; where $i=0,1,2, \cdots, I, j=0,1,2, \cdots, J$ and $n=0,1,2, \cdots$; is called a grid whose mesh size is determined by $\Delta x, \Delta y$ and $\Delta t$. The approximation to $\bar{\theta}(i \Delta x, j \Delta y, n \Delta t)$ is denoted by $\theta_{i j}^{n}$. In the same way, $\bar{u}(i \Delta x, j \Delta y, n \Delta t)$, $\bar{v}(i \Delta x, j \Delta y, n \Delta t)$, and $\bar{\rho}(i \Delta x, j \Delta y, n \Delta t)$, are denoted by $u_{i j}^{n}, v_{i j}^{n}$ and $\rho_{i j}^{n}$, respectively. The finite difference equation [4] approximating the energy equation is obtained

$$
\begin{aligned}
\frac{\theta_{i j}^{n+1}-\theta_{i i}^{n}}{\Delta t}+u_{i j}^{m}\left[\frac{\partial \theta}{\partial x}\right]_{i j}^{k} \\
=\left[\sigma_{i j}^{m}+K_{i j}^{m}\right]\left\{\frac{\theta_{i+1 j}^{k}-2 \theta_{i j}^{k}+\theta_{i-1 i}^{k}}{\Delta x^{2}}+\frac{\theta_{i j+1}^{k}-2 \theta_{i j}^{k}+\theta_{i j-1}^{k}}{\Delta y^{2}}\right\} \\
+\left\{\frac{K_{i j}^{m}}{\rho_{i j}^{m}} \frac{\rho_{i+1 j}^{m}-\rho_{i-1 j}^{m}}{\Delta x}+\frac{K_{i+1 j}^{m}-K_{i-1 j}^{m}}{2 \Delta x}\right\} \frac{\theta_{i+1 j}^{k}-\theta_{i-1 j}^{k}}{2 \Delta x} \\
+\left\{\frac{K_{i j}^{m}}{\rho_{i j}^{m}} \frac{\rho_{i j+1}^{m}-\rho_{i j-1}^{m}}{\Delta y}+\frac{K_{i j+1}^{m}-K_{i j-1}^{m}}{2 \Delta y}\right\} \frac{\theta_{i j+1}^{k}-\theta_{i j-1}^{k}}{2 \Delta y}
\end{aligned}
$$

where

$$
\left\{\begin{array}{l}
\sigma_{i j}^{m}=\frac{\lambda_{\theta}}{\rho_{i j}^{m} c_{p}} \\
k= \begin{cases}n & \text { explicit scheme } \\
n+1 & \text { implicit scheme }\end{cases} \\
m= \begin{cases}n & \text { decoupled method } \\
n+1 & \text { coupled method }\end{cases}
\end{array}\right.
$$

The decoupled method is used in our system. The time derivative term is approximated with two-point backward implicit $(k=n+1)$ time difference scheme. The diffusion terms and first order derivative terms are approximated with five-point or three-point central space difference scheme, respectively. The convection terms $u_{i j}^{n}\left[\frac{\partial \theta}{\partial x}\right]_{i j}^{k}$ and $v_{i j}^{\mathrm{n}}\left[\frac{\partial \theta}{\partial y}\right]_{i j}^{k}$, which are represented by $a\left[\frac{\partial f}{\partial h}\right]_{l}$, are approximated with following scheme:

(1) Central difference scheme

$$
a\left[\frac{\partial f}{\partial h}\right]_{l}=\frac{f_{l+1}-f_{l-1}}{2 \Delta h}
$$

(2) Two-point upwind difference scheme

$$
a\left[\frac{\partial f}{\partial h}\right]_{l}= \begin{cases}a \frac{f_{l}-f_{l-1}}{\Delta h} & \text { if } a \geq 0 \\ a \frac{f_{l+1}-f_{l}}{\Delta h} & \text { if } a<0\end{cases}
$$


(3) Three-point upwind difference scheme

$$
a\left[\frac{\partial f}{\partial h}\right]_{l}= \begin{cases}a \frac{3 f_{l}-4 f_{l-1}+f_{l-2}}{\Delta h} & \text { if } a \geq 0 \\ a \frac{-f_{l+2}+4 f_{l+1}-3 f_{l}}{\Delta h} & \text { if } a<0\end{cases}
$$

(4) Four-point downwind difference scheme I

$$
a\left[\frac{\partial f}{\partial h}\right]_{l}= \begin{cases}a \frac{f_{l+2}-2 f_{l+1}+3 f_{l}-2 f_{l-1}}{\Delta h} & \text { if } a \geq 0 \\ \frac{2 f_{l+1}-3 f_{l}+2 f_{l-1}-f_{l-2}}{\Delta h} & \text { if } a<0\end{cases}
$$

(5) Four-point dow nwind difference scheme II

$$
a\left[\frac{\partial f}{\partial h}\right]_{l}= \begin{cases}a \frac{2 f_{l+2}-3 f_{l+1}+6 f_{l}-5 f_{l-1}}{\Delta h} & \text { if } a \geq 0 \\ a \frac{5 f_{l+1}-6 f_{l}+3 f_{l-1}-2 f_{l-2}}{\Delta h} & \text { if } a<0\end{cases}
$$

Truncation errors

The truncation errors are worthy of some discussions to estimate the accuracy of numerical solutions. The estimates are obtained by Taylor series analysis. The solutions of each scheme of the difference equation of energy are equivalent to the solutions of the following differential equations.

(A) Tow-point upwind difference scheme

$$
\begin{aligned}
& \frac{\partial \bar{\theta}}{\partial t}+\vec{u} \frac{\partial \tilde{\theta}}{\partial x}+ \bar{v} \frac{\partial \bar{\theta}}{\partial y}=\left\{\left(\frac{\lambda_{\theta}}{\bar{\rho} c_{p}}+K\right)+\Delta x \frac{|\bar{u}|}{2}\right\} \frac{\partial^{2} \bar{\theta}}{\partial x^{2}} \\
&+\left\{\left(\frac{\lambda_{\theta}}{\bar{\rho} c_{p}}+K\right)+\Delta y \frac{|\bar{p}|}{2}\right\} \frac{\partial^{2} \bar{\theta}}{\partial y^{2}}+\left\{2 \frac{K}{\bar{\rho}} \frac{\partial \bar{\rho}}{\partial x}+\frac{\partial K}{\partial x}\right\} \frac{\partial \bar{\theta}}{\partial x} \\
&+\left\{2 \frac{K}{\bar{\rho}} \frac{\partial \bar{\rho}}{\partial y}+\frac{\partial K}{\partial y}\right\} \frac{\partial \bar{\theta}}{\partial y}+O(\Delta t)+O\left(\Delta x^{2}\right)+O\left(\Delta y^{2}\right) \\
& \text { as } \Delta t, \Delta x, \Delta y \rightarrow 0
\end{aligned}
$$

(B) Other difference scheme

$$
\begin{aligned}
\frac{\partial \bar{\theta}}{\partial t}+\bar{u} \frac{\partial \bar{\theta}}{\partial x}+\bar{y} \frac{\partial \bar{\theta}}{\partial y} & =\left(\frac{\lambda_{\theta}}{\bar{\rho} c_{p}}+K\right) \frac{\partial^{2} \bar{\theta}}{\partial x^{2}}+\left(\frac{\lambda_{\theta}}{\bar{\rho} c_{p}}+K\right) \frac{\partial^{2} \bar{\theta}}{\partial y^{2}} \\
& +\left\{2 \frac{K}{\bar{\rho}} \frac{\partial \bar{\rho}}{\partial x}+\frac{\partial K}{\partial x}\right\} \frac{\partial \bar{\theta}}{\partial x}+\left\{2 \frac{K}{\bar{\rho}} \frac{\partial \bar{\rho}}{\partial y}+\frac{\partial K}{\partial y}\right\} \frac{\partial \bar{\theta}}{\partial y} \\
& +O(\Delta t)+O\left(\Delta x^{2}\right)+O\left(\Delta y^{2}\right) \\
& \text { as } \Delta t, \Delta x, \Delta y \rightarrow 0
\end{aligned}
$$


The coefficient of the terms $O(\Delta t), O\left(\Delta x^{2}\right)$ and $O\left(\Delta y^{2}\right)$ involves the derivations of high order than it appears in these equations. The truncation errors are evaluated by $O(\Delta t)+O\left(\Delta x^{2}\right)+O\left(\Delta y^{2}\right)$. As the differencing of the convection terms are applied to two-point upwind difference scheme, the diffusion terms are made additions to $\Delta x|\bar{u}| / 2$ and $\Delta y|\bar{y}| / 2$ which are called numerical viscosity. Using the other difference scheme, however, the numerical viscosity does not come out. Therefore the accuracy of the numerical solutions depends only upon the time and space mesh sizes under no existence of rounding-off errors by numerical computations.

\section{Practical Stability and Spurious Oscillation}

The integration of the parabolic partial differential energy equation in time and space requires the practical stability for the finite difference method. Practical stability imposed restrictions on the size of time mesh and space meshes for the finite difference scheme, but the sizes of $\Delta t, \Delta x$ and $\Delta y$ are arbitrarily given. We obtained the practical stability conditions impose restrictions for each scherne on the mesh sizes of $\Delta t, \Delta x$ and $\Delta y$ as shown in Table 1.

We consider the accuracy of computational results for high Reynolds number because of turbulent fluid flow. The computational results have the spurious error [5] under the condition of the effective maximum cell Reynolds number ( $\left.R e^{*}\right)$ greater than 2 for the equation of motion and the effective maximum cell Peclet number $\left(P e^{*}\right)$ greater than 2 for the equation of energy because of discretizing the central difference scheme in FDM (Finite Difference Method). However as the diffusion coefficient for the two-point upwind scheme is added to the numerical viscosity, the spurious oscillation is repressed or decreased by numerical viscosity for large value of velocity. $P e^{*}$ is defined as follows;

$$
P e^{*}=\max \left\{\max _{i j k} \frac{\left|u_{i j}^{n}\right| \Delta x}{\sigma_{i j}^{n}+K_{i j}^{n}}, \max _{i j k} \frac{\left|u_{i j}^{n}\right| \Delta y}{\sigma_{i j}^{n}+K_{i j}^{n}}\right\}
$$

\section{NUMERICAL EXPERIMENTS}

We consider the transient natural convection in a fire compartment of twodimensional rectangular room (2.4 $\mathrm{m}$ height and $2.4 \mathrm{~m}$ length). Steady flat plate heat source $\left(800^{\circ} \mathrm{C}\right)$ is placed [A] at the center of the floor $(10 \mathrm{~cm}$ width) in Fig. 1 and $[B]$ at the left hand side wall (2.4 $\mathrm{m}$ length) in Fig. 2. The fluid in the fire compartment is initially motionless and at a uniform temperature of $30^{\circ} \mathrm{C}$. Initial pressure and density distribution are obtained by computation of the equation of state. The ceiling, floor and left side wall are the solid boundary and the right side is the free space boundary. The solid boundaries are assumed to be thermally adiabatic, Neumann type, except to the heating plate and the velocity on the solid boundary is assumed to be Dirichlet type non-slip condition. The boundary conditions on the free space boundary are assumed to be Neumann type condition for out-flow and Dirichlet type condition for in-flow. The simultaneous equations introduced by the implicit difference scherne are solved numerically by the sparce 
line successive over-relaxation method (SLSOR) for Poisson type equation and twopoint upwind difference scheme, and by the sparce conjugate residual II method (SCR2) for other difference scheme to reduce the computer memories of data area. Several numerical experiments were carried out on FACOM VP50 with FORTRAN 77 used double precision as follows;

[1] Estimate of the computational results with space mesh

The constant line heat source $\left(800^{\circ} \mathrm{C}\right.$ and $2.4 \mathrm{~m}$ width $)$ is located on the left side solid wall in Fig. 1. The computational domain is subdivided with the total $11 \times 11,16 \times 16,21 \times 21,31 \times 31,41 \times 41$ and $61 \times 61$ meshes corresponding to 24,16 , $12,8,6$, and $4 \mathrm{~cm}$ mesh sizes respectively. The temperature, the velocity $\bar{u}$ and $\bar{v}$ of computational results at the location $B(48 \mathrm{~cm}$ below ceiling and $48 \mathrm{~cm}$ far from heat source) and the cross section A ( $48 \mathrm{~cm}$ far from open area on free boundary) in Fig. 1 were compared each space mesh.

[2] Estimate of the computational results with different

finite difference scheme for convection terms.

For save charge computing time, the numerical computations were carried out with space mesh size $12 \mathrm{~cm}$ and time interval $10 \mathrm{msec}$ in which the heat source is located on the left side solid wall and on the floor in cases of Fig. 1. The scheme for convection term is proposed numerically.

[3] Estimate of the computational results with time interval

As the results of the estimate of the space mesh, the computational domain is subdivided into $41 \times 41$ grids corresponding to $6 \mathrm{~cm}$ mesh size in Fig. 2. The computations were carried out with time intervals which are chosen $2.5,5,7.5,10$, $15,20,30$ and $40 \mathrm{msec}$ considering truncation errors. The temperature, the velocity $\bar{u}$ and $\ddot{v}$ of computational results at the location A (center of ceiling and $48 \mathrm{~cm}$ below ceiling) and the cross section on the free boundary in Fig. 2 were compared.

\section{RESULTS and DISCUSSIONS}

[1] Estimate of the computational results with space mesh

In order to estimate the accuracy of computational results applied to two-point upwind difference, the space meshes are chosen $24,16,12,8,6$ and $4 \mathrm{~cm}$, and the time interval is fixed constant 10 msec. Fig. 3 shows the computational results of the temperature and the velocity $\bar{u}$ at the location $B$. The temperature differences and the velocity component $\bar{u}$ differences among the space meshes 4,6 and $8 \mathrm{~cm}$ in Fig. 3-a and 3-b respectively are the much same values (less than $5 \%$ errors). In Fig. 3-a, the computational results show the oscillation called "spurious oscillation". Fig. 4 shows the relationship between $R e^{*}$ from the computational results and time. This $R e^{*}$ of $4 \mathrm{~cm}$ mesh in Fig. 4 gives the smallest values less than 10 after $10 \mathrm{sec}$, so the period of spurious oscillation would give the large and the amplitude would give the small. Before $10 \mathrm{sec}$ the flow motion is numerically unstable because of initially putting the constant line heat source temperature $800^{\circ} \mathrm{C}$, so the numerical computation with $24 \mathrm{~cm}$ mesh and $10 \mathrm{msec}$ time interval was diverged and in the case of $2 \mathrm{~cm}$ and $10 \mathrm{msec}$ time interval was also diverged because the simultaneous equations for implicit method were unstable to be solve numerically by the truncation errors and rounding-off errors. Fig. 5 shows the computational 
results of the temperature at the cross section $\mathbf{A}$. The results with space mesh sizes 6 and $4 \mathrm{~cm}$ are the much same and the other mesh sizes are quite different from them. On the other hand Fig. 6 shows the temperature distribution at the cross section $A$ with time intervals 10 and $5 \mathrm{msec}$ in the case of space mesh $6 \mathrm{~cm}$, and with time intervals $10 \mathrm{msec}, 5 \mathrm{msec}$ and $2.5 \mathrm{msec}$ in the case of $4 \mathrm{~cm}$. These time intervals are given by considering truncation errors. As the results the temperature difference is about $10 \%$ errors each other. Table 2 shows the computational run time (CPU time) of 20 simulation seconds.

[2] Estimate of the computational results with different

finite difference scheme for convection terms.

Fig. 7 shows the distributions of temperature with the different scheme for convection term in the case of $10 \mathrm{msec}$ time interval and $12 \mathrm{~cm}$ mesh at the cross section of free boundary in Fig. 1. As the results the two-point upwind scheme is only quite differences among other scheme, that is, it gives under estimate because of adding the numerical viscosity. Table 3 show the CPU time until 20 simulation seconds. In above mentions the three-point upwind difference scheme for convection term applied implicit method would be better way.

[3] Estimate of the computational results with time interval

The computations were carried out for the accuracy of time interval with $6 \mathrm{~cm}$ mesh applied to three-point upwind scheme in the case of Fig. 2. The time intervals are chosen $2.5,5,7.5,10,15,20,30$, and $40 \mathrm{msec}$. In the case of $40 \mathrm{msec}$ time interval the computation miscarried due to numerical errors. It should be noted that the computations were only success the time intervals $7.5,10$, and $15 \mathrm{msec}$ by threepoint upwind difference scheme. Fig. 8 shows the temperature and the velocity $\bar{u}$ at the location $A$. Fig. 9 shows the temperature distributions at the cross section of free boundary. In these figures the results of temperature with $10 \mathrm{msec}$ to $2.5 \mathrm{msec}$ time intervals give about $5 \%$ errors each other. The results of the time interval 10 msec come to a full application of its values from above mentions.

\section{CONCLUSION.}

When the implicit method is used in field model simulations of a compartment fire, our personal point of view from computational experiments is that the time interval and space mesh should be chosen less than $10 \mathrm{msec}$ and $5 \mathrm{~cm}$ for high $R e$ and the difference scheme for the convection term should be the three-point upwind difference scheme. The better way is to take $O(\Delta t) \simeq O\left(\Delta x^{2}\right) \simeq O\left(\Delta y^{2}\right)$ and small increment. The mesh sizes, time and space, influence the accuracy from truncation errors under the condition of no rounding-off errors, and the scheme avoids errors due to numerical viscosity. They can be observed the trust in numerical results by $R e^{*}$ for the equation of motion and $P e^{*}$ for the equation of energy. $R e^{*}<20 \sim 30$ or $P e^{*}<20 \sim 30$ could be accepted from numerical experiments.

\section{REFERENCES}

[1] J.E. Fromm \& J.J. Smolderen; Numerical Solution of the Navier-Stokes Equa- 
tion at High Reynolds Number and the Problem of Discretization of Convective Derivatives; Numerical Methods in Fluid Dynamics; AGARD Lecture Series, No. $48(1972)$

[2] O.A. Ladyzhenskaya; The Mathematical Theory of Viscous Incompressible Flow; Gordon and Beach (1969)

[3] Y. Hasemi; Numerical Simulation of the Natural Convection in Fire Compartment; 2nd Joint Meeting UJNR (1976)

[4] Richtmyer \& Morton; Difference Methods for Initial Value Problems; John Wiley \& Sons (1967)

[5] S. Ono \& K. Hane; Advances in Numerical Method for Larger Sparse Set of Linear Equation No. 2 (1986)
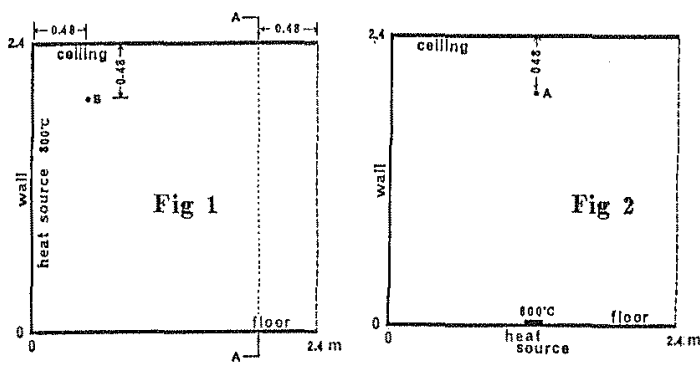

Fig. 1 The outline of computational domain

Fig. 2 The ontline of computational domain

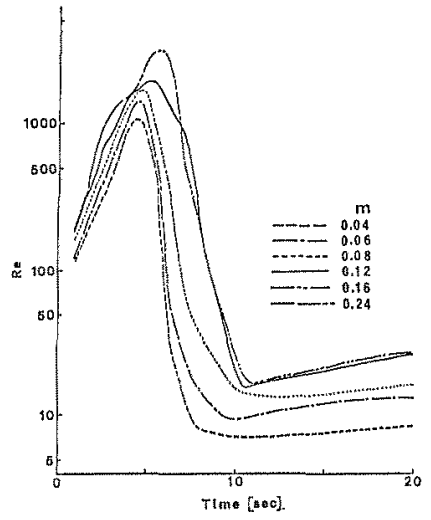

Fig. 4 Relationship between effective msximum cell $R e^{*}$ and time
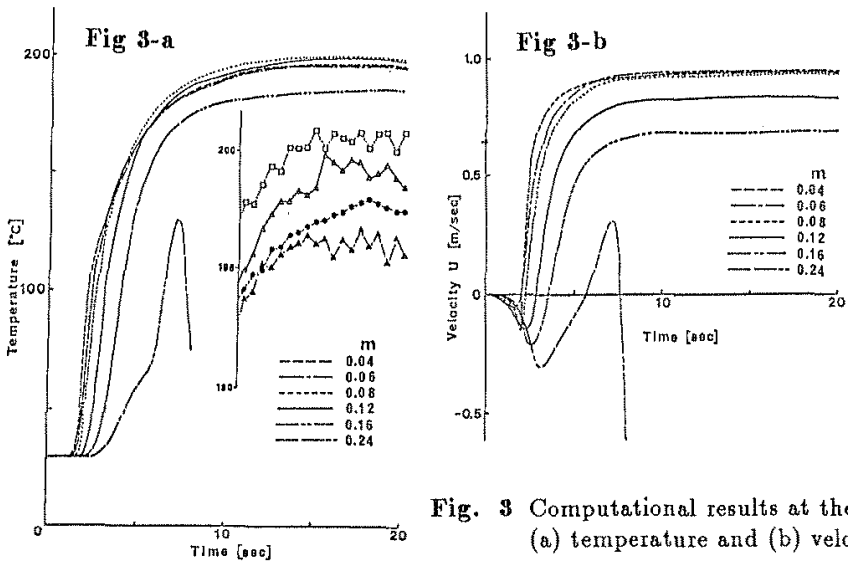

Fig. 3 Computational results at the location B;

(a) temperature and (b) velocity $u$ 


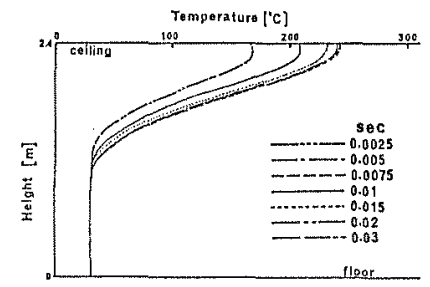

Fig. 5 Computational results of temperature at the cross section $A$.

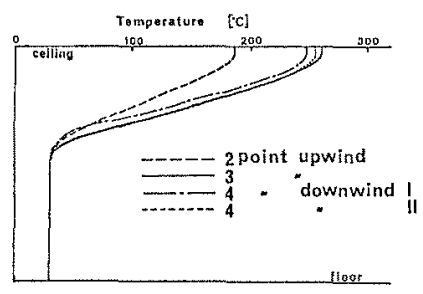

Fig. 7 Computational results of temperature at the cross section of free boundary.

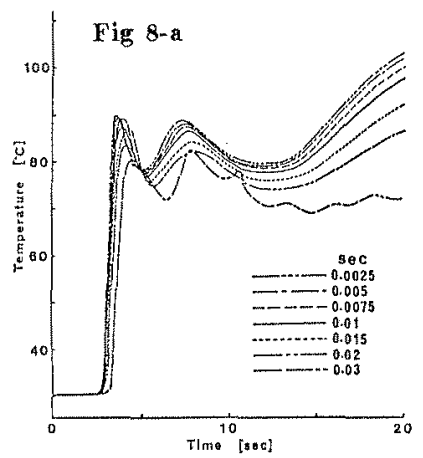

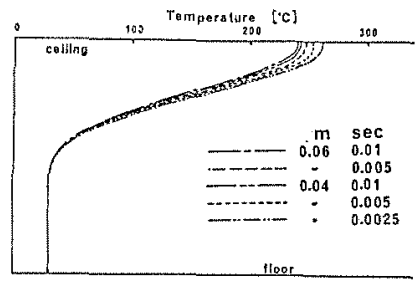

Fig. Computational results of temperature at the cross section $A$.

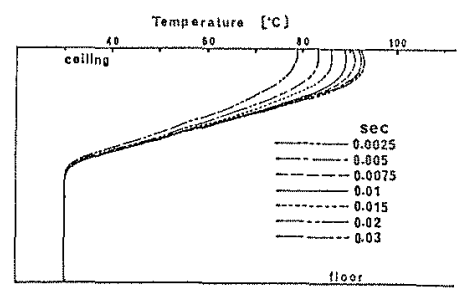

Fig. 9 Computational results of temperature at the cross section of free boundary.

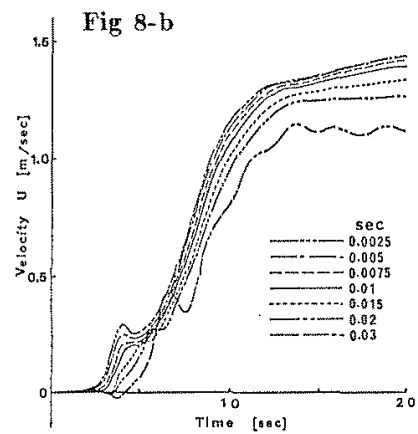

Fig. 8 Computational results at the location A.

(a) temperature and (b) velocity $u$

\begin{tabular}{|c|c|c|}
\hline & $\begin{array}{l}\text { Explicit } \\
\text { thelliod }\end{array}$ & $\begin{array}{l}\text { Iraplicit } \\
\text { method }\end{array}$ \\
\hline Centtal & {$[A]$} & always stable \\
\hline 2-point uppind & B] & always stable \\
\hline 3-point upwind & always unstable & always otable \\
\hline 1-point upwind I & always unstable & always stable \\
\hline 4-point upwind 11 & civays innstable & Almays stable \\
\hline
\end{tabular}

[A] stable if $\quad \Delta t \leq\left[\max _{i j a}\left\{2 \hat{\sigma}_{i j}^{n}\left(\frac{1}{\Delta x^{2}}+\frac{1}{\Delta y^{2}}\right)\right\}\right]^{-1}$

$$
\Delta x \leq \operatorname{man}_{i j n} \frac{2 \hat{\sigma}_{i j}^{n}}{\left|t_{i j}^{n}\right|} \text { and } \Delta y \leq \max _{i ; n} \frac{2 \hat{\sigma}_{i j}^{n} \mid}{\left|v_{i j}^{n}\right|}
$$

[B] stabie if $\Delta t \leq\left[\max _{i j \hbar}\left\{\frac{\left|u_{i n}^{n}\right|}{\Delta x}+\frac{\left|v_{i}^{n}\right|}{\Delta y}+\frac{2 \hat{\sigma}_{i j}^{n}}{\Delta x^{2}}+\frac{2 \hat{\sigma}_{i j}^{n}}{\Delta y^{2}}\right\}\right]^{-1}$

$$
\text { where } \hat{\sigma}_{i j}^{\mathrm{n}} \text { is diffusion coefficient }
$$

Table 1 Practical stability

\begin{tabular}{|c|c|c|c|}
\hline & \multicolumn{3}{|c|}{ Time interval (msec) } \\
\hline Spece mesh (cni) & 10.0 & 5.0 & 2.5 \\
\hline 16 & 0.073 & - & - \\
\hline 12 & 0.147 & - & - \\
\hline 8 & 0.418 & - & - \\
\hline 6 & 1.000 & 1.346 & - \\
\hline 4 & 5.412 & 7.360 & 11.419 \\
\hline
\end{tabular}

Table 2 CPU time

\begin{tabular}{|l|l|}
\hline \multicolumn{1}{|c|}{ Difference scheme } & \multicolumn{1}{|c|}{ ratio } \\
\hline & 0.83 (SLSOR) \\
2-point upwind difference scheme & 1.00 (SCR2) \\
\hline 3-point upwind difTerence scheme & 1.04 (SOR2) \\
\hline 4-point upwind difference scheme I & 1.54 (SOIR2) \\
\hline 4-point upwind difference scheme II & 1.34 (SCR2) \\
\hline
\end{tabular}

Table 3 CPU time 